\title{
THE NATURAL PARTIAL ORDER ON THE SEMIGROUP OF ALL TRANSFORMATIONS OF A SET THAT REFLECT AN EQUIVALENCE RELATION
}

\author{
LEI SUN ${ }^{\bowtie}$ and XIANGJUN XIN
}

(Received 13 September 2012; accepted 18 October 2012; first published online 25 January 2013)

\begin{abstract}
Let $\mathcal{T}_{X}$ be the full transformation semigroup on a set $X$ and $E$ be a nontrivial equivalence relation on $X$. Denote

$$
T_{\exists}(X)=\left\{f \in \mathcal{T}_{X}: \forall x, y \in X,(f(x), f(y)) \in E \Rightarrow(x, y) \in E\right\}
$$

so that $T_{\exists}(X)$ is a subsemigroup of $\mathcal{T}_{X}$. In this paper, we endow $T_{\exists}(X)$ with the natural partial order and investigate when two elements are related, then find elements which are compatible. Also, we characterise the minimal and maximal elements.
\end{abstract}

2010 Mathematics subject classification: primary 20M20.

Keywords and phrases: transformation semigroup, natural partial order, compatibility, minimal (maximal) elements.

\section{Introduction}

In [4] Mitsch defined a partial order on an arbitrary semigroup $S$ by

$$
a \leq b \quad \text { if and only if } a=x b=b y \text { and } a=a y \text { for some } x, y \in S^{1},
$$

and this is called the natural partial order on $S$. Later Kowol and Mitsch in [2] studied various properties of this partial order on the full transformation semigroup $\mathcal{T}_{X}$ consisting of all total transformations of an arbitrary nonempty set $X$. Then MarquesSmith and Sullivan in [3] extended some of the previous work to the semigroup $\mathcal{P}_{X}$ of all partial transformations on $X$. Sullivan in [11] investigated the partial order on the linear transformation semigroup $P(V)$ for a vector space $V$. In [10] Singha et al. considered the partial order on the partial Baer-Levi semigroup, and so on (see $[12,13])$.

This paper is supported by National Natural Science Foundation of China (No. 10971086).

(c) 2013 Australian Mathematical Publishing Association Inc. 0004-9727/2013 \$16.00 
Let $E$ be an equivalence relation on the set $X$. The subsemigroup of $\mathcal{T}_{X}$ defined by

$$
T_{E}(X)=\left\{f \in \mathcal{T}_{X}: \forall x, y \in X,(x, y) \in E \Rightarrow(f(x), f(y)) \in E\right\}
$$

was mainly studied in [5-9] and the natural partial order on the semigroup $T_{E}(X)$ was investigated in [12]. Inspired by the semigroup $T_{E}(X)$, the authors in [1] considered the semigroup

$$
T_{\exists}(X)=\left\{f \in \mathcal{T}_{X}: \forall x, y \in X,(f(x), f(y)) \in E \Rightarrow(x, y) \in E\right\}
$$

which differs greatly from the semigroup $T_{E}(X)$. The transformation $f \in T_{\exists}(X)$ reflects the equivalence relation $E$. Clearly, $T_{\exists}(X)$ is also a subsemigroup of $\mathcal{T}_{X}$ and contains the identity transformation $\operatorname{id}_{X}$ on $X$. Moreover, if $E=X \times X$, then $T_{\exists}(X)=\mathcal{T}_{X}$. If $E=\Delta=\{(x, x): x \in X\}$, then

$$
T_{\exists}(X)=\left\{f \in \mathcal{T}_{X}: f \text { is injective }\right\} .
$$

So to this extent it is regarded as a generalisation of $\mathcal{T}_{X}$.

In this paper, we assume the set $X$ is finite or infinite, the equivalence relation $E$ is nontrivial (that is, $E \neq X \times X$ and $E \neq \Delta$ ) and $X / E$, which is the partition of $X$ induced by $E$, is finite or infinite, and consider the semigroup $T_{\exists}(X)$ endowed with the natural partial order. Denote by $f g$ the transformation obtained by performing first $g$ and then $f$. Then the natural partial order can be written, for $f, g \in T_{\exists}(X)$, as

$$
f \leq g \quad \text { if and only if } f=k g=g h \text { and } f=k f \text { for some } k, h \in T_{\exists}(X) .
$$

This paper is organised as follows. In Section 2 we give a characterisation of the natural partial order on the semigroup $T_{\exists}(X)$. In Section 3 we find the elements which are compatible with the natural partial order. And in Section 4 we characterise the minimal and maximal elements.

The following lemma describes an essential property of $T_{\exists}(X)$.

Lemma 1.1 [1]. Let $f \in T_{\exists}(X)$. Then for each $A \in X / E, f(A) \subseteq \bigcup_{i \in I} B_{i}$ where I is some index set and $B_{i} \in X / E$.

\section{Characterisation}

Let $\pi(f)$ be the partition of $X$ induced by $f \in \mathcal{T}_{X}$, namely,

$$
\pi(f)=\left\{f^{-1}(y): y \in f(X)\right\} .
$$

Denote

$$
Z(f)=\{A \in X / E: A \cap f(X)=\emptyset\} .
$$

Let $\mathcal{A}, \mathcal{B}$ be two collections of subsets of $X$. If for each $A \in \mathcal{A}$, there exists some $B \in \mathcal{B}$ such that $A \subseteq B$, then $\mathcal{A}$ is said to refine $\mathcal{B}$. For $A \subseteq X$, let

$$
\overline{f(A)}=\{B \in X / E: B \cap f(A) \neq \emptyset\} .
$$


The following theorem gives a characterisation of this partial order.

Theorem 2.1. Let $f, g \in T_{\exists}(X)$. Then $f \leq g$ if and only if the following statements hold.

(1) $\pi(g)$ refines $\pi(f)$ and $|Z(g)| \leq|Z(f)|$.

(2) If $(f(x), f(y)) \in E$ for some distinct $x, y \in X$, then $(g(x), g(y)) \in E$.

(3) If $g(x) \in f(X)$ for some $x \in X$, then $f(x)=g(x)$.

(4) For each $A \in X / E$, there exists a unique $B \in X / E$ such that $f(A) \subseteq g(B)$.

Proof. Suppose that $f \leq g$. Then there exist some $k, h \in T_{\exists}(X)$ such that

$$
f=k g=g h \quad \text { and } \quad f=k f .
$$

It follows from $f=k g$ that $\pi(g)$ refines $\pi(f)$. By $f(X)=k g(X), f(X) \cap k(A)=\emptyset$ for each $A \in Z(g)$. Then there is some $B \in Z(f)$ such that $B \cap k(A) \neq \emptyset$. By $k \in T_{\exists}(X)$, $|Z(g)| \leq|Z(f)|$ and (1) holds. Let $(f(x), f(y)) \in E$ for some distinct $x, y \in X$, that is, $(k g(x), k g(y)) \in E$. Then, by $k \in T_{\exists}(X),(g(x), g(y)) \in E$ and (2) holds. Now if $g(x) \in f(X)$ for some $x \in X$, then $g(x)=f(y)$ for some $y \in X$. So

$$
f(x)=k g(x)=k f(y)=f(y)=g(x)
$$

and (3) holds. For each $A \in X / E$, let $\overline{h(A)}=\left\{B_{i}: i \in I\right\}$ where $B_{i} \in X / E$ and $I$ is some index set. Then, for each $M \in \overline{f(A)}$,

$$
f(A) \cap M=g h(A) \cap M \subseteq g\left(\bigcup_{i \in I} B_{i}\right) \cap M .
$$

By $g \in T_{\exists}(X)$, we know that $g$ does not map the different $E$-classes to the same $E$ class. So there is a unique $i \in I$ such that $f(A) \cap M \subseteq g\left(B_{i}\right) \cap M$. Write $B=B_{i}$ and then $f(A) \cap M \subseteq g(B) \cap M$. Therefore, $f(A) \subseteq g(B)$ and (4) holds.

Conversely, suppose that conditions (1)-(4) hold. Then, by $|Z(g)| \leq|Z(f)|$, there is a map

$$
\rho: \mathcal{M}=\{\bigcup A: A \in Z(g)\} \rightarrow \mathcal{N}=\{\bigcup B: B \in Z(f)\}
$$

such that $(x, y) \notin E \Rightarrow(\rho(x), \rho(y)) \notin E$ for any $x, y \in \mathcal{M}$. We define $k$ on each $E$-class $A$. There are two cases to consider.

Case 1. $A \cap g(X)=\emptyset$. For each $z \in A$, let $k(z)=\rho(z)$.

Case 2. $A \cap g(X) \neq \emptyset$. For each $z \in A \cap g(X)$, then $z=g(x)$ for some $x \in X$ and define $k(z)=f(x)$. Fix a point $z_{A} \in A \cap g(X)$ and let $k(z)=k\left(z_{A}\right)$ for each $z \in A-g(X)$. If some $x^{\prime} \in X$ satisfies $z=g\left(x^{\prime}\right)=g(x)$, then $f\left(x^{\prime}\right)=f(x)$ since $\pi(g)$ refines $\pi(f)$. Thus $k$ is well defined on $A$. Consequently, $k$ is well defined on all of $X$. Moreover, $k(A) \subseteq f(X)$.

Now we verify that $k \in T_{\exists}(X)$. Let $x \in A_{1}$ and $y \in A_{2}$ for some $\operatorname{distinct} A_{1}, A_{2} \in X / E$. We discuss three cases.

Case 1. $A_{1} \cap g(X)=\emptyset$ and $A_{2} \cap g(X)=\emptyset$. Then $(k(x), k(y))=(\rho(x), \rho(y)) \notin E$. 
Case 2. $A_{1} \cap g(X)=\emptyset$ and $A_{2} \cap g(X) \neq \emptyset$. We discuss two subcases.

Case 2.1. $y \in A_{2} \cap g(X)$. Then $k(x)=\rho(x)$ and $k(y) \in f(X)$. So $(k(x), k(y)) \notin E$.

Case 2.2. $y \in A_{2}-A_{2} \cap g(X)$. In this case $k(y)=k\left(z_{A_{2}}\right)$ where $z_{A_{2}}$ is a fixed point in $A_{2} \cap g(X)$. So $(k(x), k(y))=\left(k(x), k\left(z_{A_{2}}\right)\right) \notin E$ (by Case 2.1).

Case 3. $A_{1} \cap g(X) \neq \emptyset$ and $A_{2} \cap g(X) \neq \emptyset$. We discuss three subcases.

Case 3.1. $x \in A_{1} \cap g(X)$ and $y \in A_{2} \cap g(X)$. Then $x=g\left(x^{\prime}\right), y=g\left(y^{\prime}\right)$ for some distinct $x^{\prime}, y^{\prime} \in X$. We assert that $(k(x), k(y)) \notin E$. Indeed, if $(k(x), k(y)) \in E$, namely, $\left(f\left(x^{\prime}\right), f\left(y^{\prime}\right)\right) \in E$, then, by (2), we have $\left(g\left(x^{\prime}\right), g\left(y^{\prime}\right)\right) \in E$, that is, $(x, y) \in E$, a contradiction.

Case 3.2. $x \in A_{1}-A_{1} \cap g(X)$ and $y \in A_{2} \cap g(X)$. Then we have $k(x)=k\left(z_{A_{1}}\right)$ and $\left(k\left(z_{A_{1}}\right), k(y)\right) \notin E$ (by Case 3.1). So $(k(x), k(y)) \notin E$.

Case 3.3. $x \in A_{1}-A_{1} \cap g(X)$ and $y \in A_{2}-A_{2} \cap g(X)$. Then $k(x)=k\left(z_{A_{1}}\right), k(y)=k\left(z_{A_{2}}\right)$ and $\left(k\left(z_{A_{1}}\right), k\left(z_{A_{2}}\right)\right) \notin E$ (by Case 3.1). So $(k(x), k(y)) \notin E$.

In any case $k \in T_{\exists}(X)$. It is clear that $f=k g$. We show that $f=k f$. For each $x \in X$, by (4), there exists some $y \in X$ such that $f(x)=g(y)$ and it follows from (3) that $f(y)=g(y)$. So

$$
f(x)=f(y)=k g(y)=k f(x)
$$

which means that $f=k f$.

Finally, we define $h$ on $X$. For each $A \in X / E$ and each $x \in A$, there exists a unique $B \in X / E$ such that $y \in B$ and $f(x)=g(y)$. Define $h(x)=y$ as required. By $f, g \in T_{\exists}(X)$ and the uniqueness of the $E$-class $B$ associated with each $E$-class $A$, we have $h \in T_{\exists}(X)$. It is clear that $f=g h$. This completes the proof.

Corollary 2.2. Let $f, g \in T_{\exists}(X)$. Then the following statements hold.

(1) If $f \leq g$, then $f(X) \subseteq g(X)$.

(2) If $f \leq g$ and $f(X)=g(X)$, then $f=g$.

(3) If $f \leq g$ and $\pi(f)=\pi(g)$, then $f=g$.

Proof. (1) This follows from Theorem 2.1(4).

(2) This follows from Theorem 2.1(3).

(3) By (1), $f(X) \subseteq g(X)$. If $f(X) \subset g(X)$ (where $f(X) \subset g(X)$ means that $f(X)$ is a proper subset of $g(X))$, then take $y \in g(X)-f(X)$ and let $g(x)=y$ for some $x \in X$. So $f(x)=g\left(x^{\prime}\right)$ for some $x^{\prime} \in X\left(x^{\prime} \neq x\right)$. By Theorem 2.1(3), $f\left(x^{\prime}\right)=g\left(x^{\prime}\right)$ which implies that $f\left(x^{\prime}\right)=f(x)$. Since $\pi(f)=\pi(g)$, we have $g\left(x^{\prime}\right)=g(x)$. Observing that $g\left(x^{\prime}\right)=$ $f(x), g(x)=y$, we deduce that $f(x)=y$, a contradiction. Therefore, $f(X)=g(X)$. By (2), $f=g$.

\section{Compatibility}

A transformation $h \in T_{\exists}(X)$ is said to be strictly left compatible with the partial order if $h f<h g$ for all $f<g$. Strict right compatibility is defined dually. 
Theorem 3.1. Let $h \in T_{\exists}(X)$. Then $h$ is strictly left compatible if and only if $h$ is injective and $h(A) \subseteq B \in X / E$ for each $A \in X / E$.

Proof. Suppose that $h$ is strictly left compatible. We claim that $h$ is injective. Indeed, let $h(a)=h(b)$ for some distinct $a, b \in C \in X / E$. Assume that $C$ is a disjoint union of nonempty sets $C_{1}$ and $C_{2}$ (namely, $C=C_{1} \cup C_{2}$ and $C_{1} \cap C_{2}=\emptyset$ ) and $a \in C_{1}, b \in C_{2}$. Define $f, g: X \rightarrow X$ by

$$
f(x)=\left\{\begin{array}{ll}
a & \text { if } x \in C \\
x & \text { otherwise }
\end{array} \quad \text { and } \quad g(x)= \begin{cases}a & \text { if } x \in C_{1} \\
b & \text { if } x \in C_{2} \\
x & \text { otherwise }\end{cases}\right.
$$

respectively. Clearly, $f, g \in T_{\exists}(X)$ and $f \neq g$. It is straightforward to show $f<g$. Then $h f<h g$ and $h f(X) \subset h g(X)$. However, by the assumption $h(a)=h(b), h f(C)=h g(C)$ and $h f(D)=h g(D)$ for any other $E$-class $D$ which implies that $h f(X)=h g(X)$, a contradiction. It follows that $h$ is injective.

To verify the remaining conclusion, assume without loss of generality that $\overline{h(A)}=$ $\left\{B_{1}, B_{2}\right\}$ for some $A \in X / E$. Denote

$$
A_{1}=\left\{x \in A: h(x) \in B_{1}\right\} \quad \text { and } \quad A_{2}=\left\{x \in A: h(x) \in B_{2}\right\} .
$$

Then $A$ is a disjoint union of nonempty sets $A_{1}$ and $A_{2}$. Take $x^{\prime} \in A_{1}$ and define $f: X \rightarrow X$ by

$$
f(x)= \begin{cases}x^{\prime} & \text { if } x \in A \\ x & \text { otherwise. }\end{cases}
$$

Clearly, $f \in T_{\exists}(X), f \neq \mathrm{id}_{X}$ and $f<\operatorname{id}_{X}$. Thus $h f<h \mathrm{id}_{X}$. However, taking $y^{\prime} \in A_{2}$, we have $\left(h f\left(x^{\prime}\right), h f\left(y^{\prime}\right)\right) \in E, h \operatorname{id}_{X}\left(x^{\prime}\right) \in B_{1}, h \operatorname{id}_{X}\left(y^{\prime}\right) \in B_{2}$ which means that $\left(h f\left(x^{\prime}\right)\right.$, $\left.h f\left(y^{\prime}\right)\right) \in E$ does not imply $\left(h \operatorname{id}_{X}\left(x^{\prime}\right), h \operatorname{id}_{X}\left(y^{\prime}\right)\right) \in E$, a contradiction.

Conversely, let $f, g \in T_{\exists}(X)$ and $f<g$. Clearly, $\pi(h g)$ refines $\pi(h f)$. Write

$$
\overline{f(X)}=\left\{A_{i}: i \in I\right\} \quad \text { and } \overline{g(X)}=\left\{B_{j}: j \in J\right\},
$$

where $I, J$ are some index sets. Since $h$ maps any $E$-class to one $E$-class, let $h\left(A_{i}\right) \subseteq C_{i}$ and $h\left(B_{j}\right) \subseteq D_{j}$ for each $i \in I, j \in J$. Then $\overline{h f(X)}=\left\{C_{i}: i \in I\right\}$ and $\overline{h g(X)}=\left\{D_{j}: j \in J\right\}$. By $|Z(g)| \leq|Z(f)|$, we have $|\overline{f(X)}| \leq|\overline{g(X)}|$ and $|\overline{h f(X)}| \leq|\overline{h g(X)}|$. So $|Z(h g)| \leq|Z(h f)|$ and $h f, h g$ satisfy Theorem 2.1(1). Let $(h f(x), h f(y)) \in E$ for some distinct $x, y \in X$. Then $(f(x), f(y)) \in E$. By $f<g$, we deduce $(g(x), g(y)) \in E$. Thus $(h g(x), h g(y)) \in E$ which implies that $h f, h g$ satisfy Theorem 2.1(2). It is clear that $h f, h g$ satisfy Theorem 2.1(3). For each $A \in X / E$ and $M \in \overline{h f(A)}$, we have $h f(A) \cap M \neq \emptyset$ and there is some $N \in \overline{f(A)}$ such that $h(f(A) \cap N) \cap M \neq \emptyset$. Thus, by $f<g, f(A) \cap N \subseteq$ $g(B) \cap N$ for a unique $B \in X / E$. So it follows that

$$
h f(A) \cap M=h(f(A) \cap N) \cap M \subseteq h(g(B) \cap N) \cap M=h g(B) \cap M,
$$

and $h f(A) \subseteq h g(B)$. This means that $h f, h g$ satisfy Theorem 2.1(4). Therefore, $h f<h g$. 
Note that if $X / E$ is finite, then $|\overline{h(A)}|=1$ for each $h \in T_{\exists}(X)$ and $A \in X / E$. So Theorem 3.1 is simplified as follows.

Corollary 3.2. Let $X / E$ be finite and $h \in T_{\exists}(X)$. Then $h$ is strictly left compatible if and only if $h$ is injective.

Theorem 3.3. Let $h \in T_{\exists}(X)$. Then $h$ is strictly right compatible if and only if $h$ is surjective.

Proof. Suppose that $h$ is strictly right compatible. We assert that $h$ is surjective. Indeed, for some $A \in X / E$, let $h(A) \cap B \subset B$ for some $B \in \overline{h(A)}$. Take $a \in B-h(A) \cap B$, $b \in h(A) \cap B$ and define $f, g: X \rightarrow X$ by

$$
f(x)=\left\{\begin{array}{ll}
a & \text { if } x \in h(A) \cap B \\
x & \text { otherwise }
\end{array} \quad \text { and } \quad g(x)= \begin{cases}b & \text { if } x \in h(A) \cap B \\
x & \text { otherwise }\end{cases}\right.
$$

respectively. Then $f, g \in T_{\exists}(X)$ and $f \neq g$. To see that $f<g$, let $g(x)=g(y)$ for some distinct $x, y \in X$. Then $x, y \in h(A) \cap B$ and $f(x)=f(y)$ which means that $\pi(g)$ refines $\pi(f)$. Clearly, $|Z(g)|=|Z(f)|=0$. So $f, g$ satisfy Theorem 2.1(1). If $g(x) \in$ $f(X)=X-h(A) \cap B$ for some $x \in X$, then, by the definition of $g, f(x)=g(x)=x$ which implies that $f, g$ satisfy Theorem 2.1(3). Observing that

$$
f(B)=f((h(A) \cap B) \cup(B-h(A) \cap B))=\{a\} \cup(B-h(A) \cap B)=B-h(A) \cap B
$$

and

$$
g(B)=g((h(A) \cap B) \cup(B-h(A) \cap B))=\{b\} \cup(B-h(A) \cap B),
$$

that is, $f(B) \subset g(B)$, together with $f(C)=g(C)$ for any other $E$-class $C$, we have that $f, g$ satisfy Theorem 2.1(2) and (4). Thus $f<g$ and $f h<g h$. However,

$$
f h(A) \cap B=f(h(A) \cap B) \cap B=\{a\}
$$

and

$$
g h(A) \cap B=g(h(A) \cap B) \cap B=\{b\}, \quad g h(C) \cap B=h(C) \cap B=\emptyset
$$

where $C \in X / E(C \neq A)$, which implies that there is no $E$-class $D$ such that $f h(A) \cap B \subseteq$ $g h(D) \cap B$. So $f h, g h$ do not satisfy Theorem 2.1(4), a contradiction.

Conversely, let $f, g \in T_{\exists}(X)$ and $f<g$. Clearly, $f h, g h$ satisfy Theorem 2.1(1)-(3). For each $A \in X / E$ and $M \in \overline{f h(A)}, f h(A) \cap M \neq \emptyset$ and there is some $N \in \overline{h(A)}$ such that $f(h(A) \cap N) \cap M \neq \emptyset$. Since $h$ is surjective, $h(A) \cap N=N$. Then $f(N) \cap M \subseteq$ $g(C) \cap M$ for a unique $C \in X / E$. So there is a unique $B \in X / E$ such that $h(B) \cap C=C$. It follows that

$$
\begin{aligned}
f h(A) \cap M=f(h(A) \cap N) \cap M=f(N) \cap M \subseteq g(C) \cap M & =g(h(B) \cap C) \cap M \\
& =g h(B) \cap M,
\end{aligned}
$$

that is, $f h(A) \subseteq g h(B)$, which means that $f h, g h$ satisfy Theorem 2.1(4). Therefore, $f h<g h$. 


\section{Minimal and maximal elements}

We begin by determining the minimal elements of $T_{\exists}(X)$.

Theorem 4.1. Let $f \in T_{\exists}(X)$. Then $f$ is minimal if and only if for each $A \in X / E$, $|f(A) \cap M|=1$ for each $M \in \overline{f(A)}$.

Proof. The sufficiency is clear, so we only show the necessity. If $|f(A) \cap M| \geq 2$, denote $A^{\prime}=\{x \in A: f(x) \in M\}$, then take $a \in f(A) \cap M$ and define

$$
g(x)= \begin{cases}a & \text { if } x \in A^{\prime} \\ f(x) & \text { otherwise }\end{cases}
$$

Clearly, $g \in T_{\exists}(X), g \neq f$ and $g<f$, which leads to a contradiction.

Before characterising the maximal elements of $T_{\exists}(X)$ we need some terminology. For a transformation $f \in T_{\exists}(X)$ and $A \in X / E$, we say that $\left.f\right|_{A}$ is defect-divided if $A$ is a disjoint union of nonempty sets $A_{1}$ and $A_{2}$ such that $\left.f\right|_{A_{1}}$ is not injective, $f(A) \cap M=M$ for each $M \in \overline{f\left(A_{1}\right)}$ and $\left.f\right|_{A_{2}}$ is injective, $f(A) \cap N \subset N$ for some $N \in \overline{f\left(A_{2}\right)}$. And we say that $\left.f\right|_{A}$ is surjection-divided if $\left.f\right|_{A}$ is not injective and $f(A) \cap M=M$ for each $M \in \overline{f(A)}$.

Theorem 4.2. Let $f \in T_{\exists}(X)$. Then $f$ is maximal if and only if one of the following statements holds.

(1) $f$ is injective or surjective.

(2) There is some E-class A such that $\left.f\right|_{A}$ is defect-divided. For any other E-class $B$, either $\left.f\right|_{B}$ is surjection-divided or $\left.f\right|_{B}$ is injective.

(3) There are some distinct $A, B \in X / E$ such that $\left.f\right|_{A}$ is surjection-divided and $\left.f\right|_{B}$ is injective and $f(B) \cap N \subset N$ for some $N \in \overline{f(B)}$. For any other E-class $C,\left.f\right|_{C}$ is injective and $f(C) \cap N^{\prime}=N^{\prime}$ for each $N^{\prime} \in \overline{f(C)}$.

Proof. Let $f$ be maximal. Suppose to the contrary that none of (1)-(3) holds. Assume that $\left.f\right|_{A}$ is not injective for some $A \in X / E$. Then we claim that $\left.f\right|_{A}$ is surjection-divided. Indeed, if $f(A) \cap M \subset M$ for some $M \in \overline{f(A)}$, let $A$ be a disjoint union of nonempty sets $A_{1}$ and $A_{2}$ with the property that $\left.f\right|_{A_{1}}$ is not injective and $\left.f\right|_{A_{2}}$ is injective. Then $M \notin \overline{f\left(A_{1}\right)}$. Otherwise, let $f\left(x_{1}\right)=f\left(x_{2}\right) \in M^{\prime}$ for some distinct $x_{1}, x_{2} \in A_{1}$ and take $a \in M-f(A) \cap M$. Then define $g: X \rightarrow X$ by

$$
g(x)= \begin{cases}a & \text { if } x=x_{1} \\ f(x) & \text { otherwise }\end{cases}
$$

Clearly, $g \in T_{\exists}(X), g \neq f$. It is straightforward to show that $f<g$. So $f$ is not maximal, a contradiction. It follows that $M \in \overline{f\left(A_{2}\right)}$. This also means that $f(A) \cap N=N$ for each $N \in \overline{f\left(A_{1}\right)}$. Thus $\left.f\right|_{A}$ is defect-divided, a contradiction. It follows that $\left.f\right|_{A}$ is surjection-divided. On the other hand, since $f$ is not surjective, let $f(B) \cap C \subset C$ for some $B, C \in X / E(B \neq A)$. We assert that $\left.f\right|_{B}$ is injective. Indeed, if $\left.f\right|_{B}$ is not injective, then let $B$ be a disjoint union of nonempty sets $B_{1}$ and $B_{2}$ with the property that $\left.f\right|_{B_{1}}$ 
is not injective and $\left.f\right|_{B_{2}}$ is injective. By the above approach, we deduce that $\left.f\right|_{B}$ is defect-divided, a contradiction. Thus $\left.f\right|_{B}$ is injective. Hence we find two $E$-classes $A, B$ with the property that $\left.f\right|_{A}$ is surjection-divided and $\left.f\right|_{B}$ is injective, $f(B) \cap C \subset C$, a contradiction.

Conversely, let $f \leq g$. There are three cases to consider.

Case 1. $f$ is injective or surjective. If $f$ is injective, then $\pi(f)=\pi(g)$. By Corollary 2.2(3), $f=g$. So $f$ is maximal. And if $f$ is surjective, then $f(X)=g(X)$. By Corollary 2.2(2), $f=g$. So $f$ is also maximal.

Case 2. $f$ satisfies (2). Let $A$ be a disjoint union of nonempty sets $A_{1}$ and $A_{2}$ such that $\left.f\right|_{A_{1}}$ is not injective, $f(A) \cap M=M$ for each $M \in \overline{f\left(A_{1}\right)}$ and that $\left.f\right|_{A_{2}}$ is injective, $f(A) \cap N \subset N$ for some $N \in \overline{f\left(A_{2}\right)}$. Since $f \leq g$, by Theorem 2.1(4), for each $M \in \overline{f\left(A_{1}\right)}$, there exists a unique $A^{\prime} \in X / E$ such that

$$
M=f(A) \cap M \subseteq g\left(A^{\prime}\right) \cap M \subseteq M
$$

which implies that $f(A) \cap M=g\left(A^{\prime}\right) \cap M=M$. So if $g(x) \in M$ for some $x \in A^{\prime}$, then $g(x) \in g\left(A^{\prime}\right) \cap M=f(A) \cap M$. According to Theorem 2.1(3), $f(x)=g(x)$ and $f(x) \in$ $f(A) \cap M$ which implies that $A^{\prime}=A$. This also means that $f\left(A_{1}\right)=g\left(A_{1}\right)$. Moreover, by Corollary 2.2(3), $f\left(A_{2}\right)=g\left(A_{2}\right)$. It follows that $f(A)=g(A)$. For any other $E$-class $B$, we also have $f(B)=g(B)$. Hence $f(X)=g(X)$ and $f=g$. Therefore, $f$ is maximal.

Case 3. $f$ satisfies (3). Then for each $M \in \overline{f(A)}$ there exists a unique $A^{\prime} \in X / E$ such that

$$
M=f(A) \cap M \subseteq g\left(A^{\prime}\right) \cap M \subseteq M .
$$

Similarly to Case 2, we deduce that $A^{\prime}=A$ and $f(A)=g(A)$. By Corollary 2.2(3) again, $f(B)=g(B)$ and $f(C)=g(C)$ as well. Thus $f(X)=g(X)$. So $f=g$ and $f$ is maximal.

To illustrate the maximal elements of Theorem 4.2(2) and (3), we present two examples.

Example 4.3. Let $X=\{1,2, \ldots\}$ and $E=\bigcup_{i=1}^{\infty}\left(A_{i} \times A_{i}\right)$ where $A_{1}=\{1,2,3, \ldots, 10\}$, $A_{2}=\{11,12\}, A_{3}=\{13,14,15\}, A_{4}=\{16,17,18,19\}, A_{5}=\{20,21,22,23,24\}, \ldots$

Let $f \in T_{\exists}(X)$ satisfy

$$
\left.f\right|_{A_{1}}=\left(\begin{array}{llllllllll}
1 & 2 & 3 & 4 & 5 & 6 & 7 & 8 & 9 & 10 \\
11 & 12 & 11 & 13 & 13 & 14 & 15 & 17 & 19 & 16
\end{array}\right)
$$

and $\left.f\right|_{A_{i}}$ be injective, $f\left(A_{i}\right) \subset A_{i+3}(i \geq 2)$. Clearly, $A_{1}=\{1,3,4,5\} \cup\{2,6,7,8,9,10\}$, $f(1)=f(3)=11 \in A_{2}, f(4)=f(5)=13 \in A_{3}, f\left(A_{1}\right) \cap A_{2}=A_{2}, f\left(A_{1}\right) \cap A_{3}=A_{3}$, $f\left(A_{1}\right) \cap A_{4} \subset A_{4}$. Then $\left.f\right|_{A_{1}}$ is defect-divided. Moreover, $\left.f\right|_{A_{i}}$ is injective $(i \geq 2)$. Then $f$ is a maximal element of the kind belonging to Theorem 4.2(2). 
Example 4.4. Let $X=\{1,2, \ldots, 18\}$ and $E=\bigcup_{i=1}^{4}\left(A_{i} \times A_{i}\right)$ where $A_{1}=\{1,2,3\}, A_{2}=$ $\{4,5,6,7\}, A_{3}=\{8,9,10,11,12\}$ and $A_{4}=\{13,14,15,16,17,18\}$. Let

$$
f=\left(\begin{array}{llllllllllllllllll}
1 & 2 & 3 & 4 & 5 & 6 & 7 & 8 & 9 & 10 & 11 & 12 & 13 & 14 & 15 & 16 & 17 & 18 \\
5 & 4 & 6 & 1 & 3 & 1 & 2 & 15 & 13 & 14 & 18 & 17 & 8 & 9 & 9 & 10 & 11 & 12
\end{array}\right) .
$$

Clearly, $f \in T_{\exists}(X)$ and $\left.f\right|_{A_{1}}$ is injective, $f\left(A_{1}\right) \subset A_{2}$ and $\left.f\right|_{A_{2}}$ is surjection-divided, $\left.f\right|_{A_{3}}$ is injective, $f\left(A_{3}\right) \subset A_{4}$ and $\left.f\right|_{A_{4}}$ is surjection-divided. Then $f$ is a maximal element of the kind belonging to Theorem 4.2(3).

As a consequence of Theorem 4.2, we have the following conclusion.

Corollary 4.5. Let $f \in T_{\exists}(X)$. Then the following statements hold.

(1) If $X$ is finite and all E-classes have the same size, then $f$ is maximal if and only if $f$ is a permutation preserving $E$.

(2) If $X / E$ is finite, then $f$ is maximal if and only if $f$ is either injective, or surjective, or there are some distinct $A, B \in X / E$ such that $\left.f\right|_{A}$ is surjection-divided and $\left.f\right|_{B}$ is injective and $f(B) \cap N \subset N$ for some $N \in \overline{f(B)}$, and for any other E-class $C$, $\left.f\right|_{C}$ is injective and $f(C) \cap M=M$ for each $M \in \overline{f(C)}$.

By the way, if $X / E$ is infinite, then there may be a maximal element of the kind belonging to both Theorem 4.2(2) and (3). Even if $X / E$ is finite and all $E$-classes have the same size, then there may be a maximal element of the kind belonging to Theorem 4.2(3).

Example 4.6. Let $X=\{1,2, \ldots\}$ and $E=\bigcup_{i=1}^{3}\left(A_{i} \times A_{i}\right)$, where $A_{1}=\{1,4,7, \ldots\}, A_{2}=$ $\{2,5,8, \ldots\}$ and $A_{3}=\{3,6,9, \ldots\}$. Choose

$$
f(x)= \begin{cases}3 n+3 & \text { if } x=3 n \\ 3 n-1 & \text { if } x=3 n+2 \\ x & \text { otherwise }\end{cases}
$$

where $n$ is a natural number. Clearly, $f \in T_{\exists}(X)$. Then $\left.f\right|_{A_{1}}$ is injective, $f\left(A_{1}\right)=$ $A_{1},\left.f\right|_{A_{2}}$ is surjection-divided $\left(f(2)=f(5)=2, f\left(A_{2}\right) \cap A_{2}=A_{2}\right)$ and $\left.f\right|_{A_{3}}$ is injective, $f\left(A_{3}\right) \subset A_{3} \quad\left(3 \notin f\left(A_{3}\right)\right)$. So $f$ is a maximal element of the kind belonging to Theorem 4.2(3).

\section{Acknowledgement}

We would like to thank the referee for valuable suggestions and comments which helped to improve the presentation of this paper.

\section{References}

[1] L. Z. Deng, J. W. Zeng and T. J. You, 'Green's relations and regularity for semigroups of transformations that preserve reverse direction equivalence', Semigroup Forum 83 (2011), 489498. 
[2] G. Kowol and H. Mitsch, 'Naturally ordered transformation semigroups', Monatsh. Math. 102 (1986), 115-138.

[3] M. P. O. Marques-Smith and R. P. Sullivan, 'Partial orders on transformation semigroups', Monatsh. Math. 140 (2003), 103-118.

[4] H. Mitsch, 'A natural partial order for semigroups', Proc. Amer. Math. Soc. 97 (1986), 384-388.

[5] H. S. Pei, 'Equivalences, $\alpha$-semigroups and $\alpha$-congruences', Semigroup Forum 49 (1994), 49-58.

[6] H. S. Pei, 'A regular $\alpha$-semigroup inducing a certain lattice', Semigroup Forum 53 (1996), 98-113.

[7] H. S. Pei, 'Some $\alpha$-semigroups inducing certain lattices', Semigroup Forum 57 (1998), 48-59.

[8] H. S. Pei, 'On the rank of the semigroup $T_{E}(X)$ ', Semigroup Forum 70 (2005), 107-117.

[9] H. S. Pei, 'Regularity and Green's relations for semigroups of transformations that preserve an equivalence', Comm. Algebra 33 (2005), 109-118.

[10] B. Singha, J. Sanwong and R. P. Sullivan, 'Partial order on partial Baer-Levi semigroups', Bull. Aust. Math. Soc. 81 (2010), 197-206.

[11] R. P. Sullivan, 'Partial orders on linear transformation semigroups', Proc. Roy. Soc. Edinburgh Sect. A 135 (2005), 413-437.

[12] L. Sun, H. S. Pei and Z. X. Cheng, 'Naturally ordered transformation semigroups preserving an equivalence', Bull. Aust. Math. Soc. 78 (2008), 117-128.

[13] L. Sun and L. M. Wang, 'Natural partial order in semigroups of transformations with invariant set', Bull. Aust. Math. Soc. 87 (2013), 94-107.

LEI SUN, School of Mathematics and Information Science, Henan Polytechnic University, Henan, Jiaozuo 454003, PR China e-mail: sunlei97@163.com

XIANGJUN XIN, Department of Mathematics and Information Science, Zhengzhou University of Light Industry, Henan, Zhengzhou 450002, PR China e-mail: xin_xiang_jun@126.com 